\title{
NONCOMMUTATIVE VERSIONS OF SOME CLASSICAL BIRATIONAL TRANSFORMATIONS.
}

\author{
DENNIS PRESOTTO AND MICHEL VAN DEN BERGH
}

\begin{abstract}
In this paper we generalize some classical birational transformations to the non-commutative case. In particular we show that 3-dimensional quadratic Sklyanin algebras (non-commutative projective planes) and 3-dimensional cubic Sklyanin algebras (non-commutative quadrics) have the same function field. In the same vein we construct an analogue of the Cremona transform for non-commutative projective planes.
\end{abstract}

\section{Contents}

1. Introduction

2. Reminder on AS-regular $\mathbb{Z}$-algebras

3. Non-commutative geometry

4. Construction of the subalgebra $D$ of $\check{A}^{(2)}$

5. Analysis of $D_{Y}$

6. Showing that $D$ is AS-regular

7. Non-commutative function fields

8. Relation with non-commutative blowing up References

\section{INTRODUCTION}

Below $k$ is an algebraically closed field. Artin-Schelter regular algebras were introduced in [1] and subsequently classified in dimension three [1, 3, 8]. Throughout we will only consider three-dimensional AS-regular algebras generated in degree one. For such algebras $A$ there are two possibilities:

(1) $A$ is generated by three elements satisfying three quadratic relations (the "quadratic case"). In this case $A$ has Hilbert series $1 /(1-t)^{3}$, i.e. the same Hilbert series as a polynomial ring in three variables.

(2) $A$ is generated by two elements satisfying two cubic relations (the "cubic case"). In this case $A$ has Hilbert series $1 /(1-t)^{2}\left(1-t^{2}\right)$.

For use below we define $(r, s)$ to be respectively the number of generators of $A$ and the degrees of the relations. Thus $(r, s)=(3,2)$ or $(2,3)$ depending on whether $A$ is quadratic or cubic.

The first author is an aspirant of the FWO, the second author is a senior researcher of the FWO. 
If $B=k+B_{1}+B_{2}+\ldots$ is an $\mathbb{N}$-graded ring satisfying suitable conditions then we can associate a non-commutative scheme $\operatorname{Proj} B$ to it whose category of quasi-coherent sheaves is defined to be $\mathrm{QGr}(B) \stackrel{\text { def }}{=} \operatorname{Gr}(B) / \operatorname{Tors}(B)$ where $\operatorname{Gr}(B)$ is the category of right $B$-modules and $\operatorname{Tors}(B)$ is the category graded right $B$ modules that have locally right bounded grading [5]. When $A$ is a quadratic three-dimensional AS-regular algebra then $\operatorname{Proj} A$ may be thought off as a noncommutative plane. Similarly if $A$ is cubic then $\operatorname{Proj} A$ may be viewed as a noncommutative quadric. The rationale for this is explained in [23].

The classification of three-dimensional AS-regular algebras $A$ is in terms of suitable geometric data $(Y, \sigma, \mathcal{L})$ where $Y$ is a $k$-scheme, $\sigma$ is automorphism of $Y$ and $\mathcal{L}$ is a line bundle on $Y$.

More precisely: in the quadratic case $Y$ is either $\mathbb{P}^{2}$ (the "linear case") or $Y$ is embedded as a divisor of degree 3 in $\mathbb{P}^{2}$ (the "elliptic case") and $\mathcal{L}$ is the restriction of $\mathcal{O}_{\mathbb{P}^{2}}(1)$. In the cubic case $Y$ is either $\mathbb{P}^{1} \times \mathbb{P}^{1}$ (the "linear case") or $Y$ is embedded as a divisor of bidegree $(2,2)$ in $\mathbb{P}^{1} \times \mathbb{P}^{1}$ (the "elliptic case") and $\mathcal{L}$ is the restriction of $\mathcal{O}_{\mathbb{P}^{1} \times \mathbb{P}^{1}}(1,0)$. The geometric data must also satisfy an additional numerical condition which we will not discuss here.

Starting from the geometric data $(Y, \sigma, \mathcal{L})$ we construct a so-called "twisted homogeneous coordinate ring" $B=B(Y, \sigma, \mathcal{L})$. It is an $\mathbb{N}$-graded ring such that

$$
B_{n}=\Gamma\left(Y, \mathcal{L} \otimes \mathcal{L}^{\sigma} \otimes \ldots \otimes \mathcal{L}^{\sigma^{n-1}}\right)
$$

with product $a \cdot b=a \otimes b^{\sigma^{n}}$ for $|a|=n$. The corresponding AS-regular algebra $A=A(Y, \sigma, \mathcal{L})$ is obtained from $B$ by dropping all relations in degree $>s$. By virtue of the construction there is a graded surjective $k$-algebra homomorphism $A \rightarrow B$ and this is an isomorphism in the linear case and it has a kernel generated by a normal element $g$ in degree $s+1$ in the elliptic case.

According to [4] there is an equivalence of categories $\mathrm{QGr}(B) \cong \mathrm{Q} \operatorname{ch}(Y)$. In our current language this can be written as

$$
\operatorname{Proj} B \cong Y
$$

So the non-commutative scheme $X=\operatorname{Proj} A$ contains the commutative scheme $Y$ (via the surjection $A \rightarrow B$ ). In the linear case $X=Y$, and in the quadratic case $Y$ is a so-called "divisor" in $X[22$, Section 3.6].

If $Y$ is a smooth elliptic curve, $\sigma$ is a translation such that $\sigma^{s+1} \neq$ id and $\mathcal{L}$ is a line bundle of degree $r$ then we call the corresponding AS-regular algebra a Sklyanin algebra. In that case the normal element $g$ is actually central. Since any two line bundles of the same degree on a smooth elliptic curve are related by a translation, which necessarily commutates with $\sigma$, it is easy to see that the resulting Sklyanin algebra depends up to isomorphism only on $(E, \sigma)$. So we sometimes drop $\mathcal{L}$ from the notation. Furthermore $\operatorname{Proj} A$ does not change if we compose $\sigma$ with a translation by a point of order $s+1$ (See for example $[2, \S 8]$ ). In other words $\operatorname{Proj} A$ depends only on $\sigma^{s+1}$.

A three-dimensional AS-regular algebra $A$ is a noetherian domain and in particular it has a graded field of fractions $\operatorname{Frac}(A)$ in which we invert all non-zero homogeneous elements of $A$. The part of degree zero $\operatorname{Frac}_{0}(A)$ of $\operatorname{Frac}(A)$ will be called the function field of $\operatorname{Proj} A$.

In this note we prove the following result announced in [20]. A similar result by Rogalski-Sierra-Stafford was announced in [18]. 
Theorem 1.1. If $A, A^{\prime}$ are a cubic and a quadratic Sklyanin algebra respectively with geometric data $(Y, \sigma)$ and $(Y, \psi)$ such that $\sigma^{3}=\psi^{4}$. Then $\operatorname{Proj} A$ and $\operatorname{Proj} A^{\prime}$ have the same function field.

The proof of this result is geometric. In the commutative case the passage from $\mathbb{P}^{1} \times \mathbb{P}^{1}$ to $\mathbb{P}^{2}$ goes by blowing up a point $p$ and then contracting the strict transforms of the two rulings through this point. One may short circuit this construction by considering a suitable linear system on $\mathbb{P}^{1} \times \mathbb{P}^{1}$ with base point in $p$. It is this construction that we generalize first. To do this we have to step outside the category of graded algebras and work in the slightly larger category of $\mathbb{Z}$-algebras (additive categories whose objects are indexed by $\mathbb{Z}$, see $\S 2$ below).

So what we will actually do is the following: let $A$ be a cubic Sklyanin algebra and let $A^{(2)}$ be its 2 -Veronese with the corresponding $\mathbb{Z}$-algebra being denoted by $\check{A}^{(2)}$. Associated to a point $p \in Y$ we will construct a sub-ZZ-algebra $D$ of $\breve{A}^{(2)}$ which is 3dimensional quadratic Artin-Schelter $\mathbb{Z}$-algebra in the sense of [23]. Again invoking [23] this $\mathbb{Z}$-algebra must correspond to a 3-dimensional quadratic Artin-Schelter graded algebra $A^{\prime}$. It will turn out that the geometric data of $A$ and $A^{\prime}$ are related as in Theorem 1.1. Note that the use of $\mathbb{Z}$-algebras is essential here as there is no direct embedding $A^{\prime} \hookrightarrow A^{(2)}$ of graded rings.

Another classical birational transformation is the so-called "Cremona transform". It is obtained by blowing up the tree vertices of a triangle and then contracting the sides. In this note we will also show that the Cremona transform has a non-commutative version and that it is yields an automorphism of the function field of a three-dimensional quadratic Sklyanin algebra. The properties of this automorphism will be discussed elsewhere.

In $\S 8$ we explain how in the non-commutative case the approach via linear systems is related to the blowup construction introduced in [22].

Remark 1.2. A more ring-theoretic approach to blowups of noncommutative surfaces was taken by Rogalski-Sierra-Stafford in [15]. They also used this technique in their companion paper [14] to classify certain orders in a generic 3-dimensional Sklyanin algebra.

Remark 1.3. Cubic 3-dimensional Artin-Schelter regular algebras are a special case of the non-commutative quadrics introduced in [23]. Theorem 1.1 generalizes to such quadrics but the proof becomes slightly more technical. For this reason we have chosen to write down the proof of Theorem 1.1 separately.

\section{REMiNDER ON AS-REgUlAR $\mathbb{Z}$-ALGEBRAS}

For background material on $\mathbb{Z}$-algebras see [17] and also sections 3 and 4 of [23]. Recall that a $(k$-) $\mathbb{Z}$-algebra is defined as a $k$-algebra $A$ (without unit) with a decomposition $A=\bigoplus_{(m, n) \in \mathbb{Z}^{2}} A_{m, n}$ such that the multiplication satisfies $A_{m, n} A_{n, j} \subset$ $A_{m, j}$ and $A_{m, n} A_{i, j}=0$ if $n \neq i$. Moreover we require the existence of local units $e_{n} \in A_{n n}$ satisfying $e_{m} x=x=x e_{n}$ whenever $x \in A_{m, n}$. The category of $\mathbb{Z}$-algebras is denoted by $\operatorname{Alg}(\mathbb{Z})$. Every graded $k$-algebra $A$ gives rise to a $\mathbb{Z}$-algebra $\check{A}$ via $\check{A}_{m, n}=A_{n-m}$. Most graded notions have a natural $\mathbb{Z}$-algebra counterpart. For example we say that $A \in \operatorname{Alg}(\mathbb{Z})$ is positively graded if $A_{m, n}=0$ whenever $m>n$. 
A $\mathbb{Z}$-algebra over $k$ is said to be connected, if it is positively graded, each $A_{m, n}$ is finite dimensional over $k$ and $A_{m, m} \cong k$ for all $m$.

If $A \in \operatorname{Alg}(\mathbb{Z})$ then we say $M$ is a graded right- $A$-module if it is a module in the usual sense together with a decomposition $M=\oplus_{n} M_{n}$ satisfying $M_{m} A_{m, n} \subset M_{n}$ and $M_{m} A_{i, n}=0$ if $i \neq m$. We denote the category of graded $A$-modules by $\operatorname{Gr}(A)$. (Obviously $\operatorname{Gr}(A)=\operatorname{Gr}(\check{A})$ if $A$ is a graded ring.) If $A$ is a connected $\mathbb{Z}$-algebra over $k$ we denote the graded $A$-modules $P_{n, A}=e_{n} A$ and $S_{n, A} \cong k$ is the unique simple quotient of $P_{n, A}$.

Definition 2.1. A $\mathbb{Z}$-algebra $A$ over $k$ is said to be AS-regular if the following conditions are satified:

(1) $A$ is connected

(2) $\operatorname{dim}_{k}\left(A_{m, n}\right)$ is bounded by a polynomial in $n-m$

(3) The projective dimension of $S_{n, A}$ is finite and bounded by a number independent of $n$

(4) $\forall n \in \mathbb{N}: \sum_{i, j} \operatorname{dim}_{k}\left(\operatorname{Ext}_{\operatorname{Gr}(A)}^{i}\left(S_{j, A}, P_{n, A}\right)\right)=1$

It is immediate that if a graded algebra $A$ is AS-regular in the sense of [3], then $\check{A}$ is AS-regular in the above sense.

$\mathbb{Z}$-algebra analogues of three dimensional quadratic and cubic Artin-Schelter regular algebras were classified in [23] (following [6] in the quadratic case). We will describe the quadratic case as this is the only case we will need. In this case the classification is in terms of triples $\left(Y, \mathcal{L}_{0}, \mathcal{L}_{1}\right)$ where $Y$ is either a (possibly singular, non-reduced) curve of arithmetic genus 1 (the "elliptic case") or $Y=\mathbb{P}^{2}$ (the "linear case") and $\mathcal{L}_{0}, \mathcal{L}_{1}$ are line bundles of degree 3 on $Y$ such that $\mathcal{L}_{0} \nsucceq \mathcal{L}_{1}$ in the elliptic case and $\mathcal{L}_{0}=\mathcal{L}_{1}=\mathcal{O}_{\mathbb{P}^{2}}(1)$ in the linear case. The triple must satisfy some other technical conditions which are however vacuous in the case that $Y$ is a smooth elliptic curve.

To construct a $\mathbb{Z}$-algebra from this data we first introduce the "elliptic helix" $\left(\mathcal{L}_{i}\right)_{i \in \mathbb{Z}}$ associated to $\left(\mathcal{L}_{0}, \mathcal{L}_{1}\right)$. This is a collection of line bundles satisfying

$$
\mathcal{L}_{i} \otimes_{\mathcal{O}_{Y}} \mathcal{L}_{i+1}^{-2} \otimes_{\mathcal{O}_{Y}} \mathcal{L}_{i+2}=\mathcal{O}_{Y}
$$

We put $V_{i}=H^{0}\left(Y, \mathcal{L}_{i}\right)$ and

$$
R_{i}=\operatorname{ker}\left(H^{0}\left(Y, \mathcal{L}_{i}\right) \otimes H^{0}\left(Y, \mathcal{L}_{i+1}\right) \rightarrow H^{0}\left(Y, \mathcal{L}_{i} \otimes_{\mathcal{O}_{Y}} \mathcal{L}_{i+1}\right)\right)
$$

By definition the quadratic AS-regular $\mathbb{Z}$-algebra $A=A\left(Y, \mathcal{L}_{0}, \mathcal{L}_{1}\right)$ associated to $\left(Y, \mathcal{L}_{0}, \mathcal{L}_{1}\right)$ is generated by $V_{i}\left(=A_{i, i+1}\right)$ subject to the relations $R_{i} \subset V_{i} \otimes V_{i+1}$. The "Hilbert function" of $A$ is

$$
\operatorname{dim} A_{m, m+a}= \begin{cases}\frac{(a+1)(a+2)}{2} & \text { if } a \geqslant 0 \\ 0 & \text { if } a<0\end{cases}
$$

Using the line bundles $\left(\mathcal{L}_{i}\right)_{i}$ be may define a $\mathbb{Z}$-algebra analogue $B=B\left(Y,\left(\mathcal{L}_{i}\right)_{i}\right)$ of a twisted homogeneous coordinate ring (see the introduction) where

$$
B_{m, n}=\Gamma\left(Y, \mathcal{L}_{m} \otimes \ldots \otimes \mathcal{L}_{n-1}\right)
$$

If $A$ is the 3 -dimensional AS-regular $\mathbb{Z}$-algebra constructed above then there is a surjective map

$$
\phi: A \rightarrow B
$$


where $A$ is obtained from $B$ by dropping all relations in degree $(m, n)$ for $n \geqslant$ $m+s+1$.

If $A$ is 3 -dimensional quadratic AS-regular algebra with geometric data $(Y, \sigma, \mathcal{L})$ then the elliptic helix corresponding to $\check{A}$ is $\left(\mathcal{L}^{\sigma^{i}}\right)_{i}$. This follows immediately from the construction of $A$ from $(Y, \sigma, \mathcal{L})$ as given in [3] (see the introduction for an outline).

\section{Non-commutative Geometry}

It will be convenient to use the formalism of non-commutative geometry used in [22] which we summarize here. For more details we refer to loc. cit.. See also [19]. We will change the terminology and notations slightly to be more compatible with current conventions.

For us a non-commutative scheme will be a Grothendieck category (i.e. an abelian category with a generator and exact filtered colimits). To emphasize that we think of non-commutative schemes as geometric objects, we denote them by roman capitals $X, Y, \ldots$ When we refer to the category represented by a non-commutative scheme $X$ then we write $\mathrm{Q} \operatorname{ch}(X)$.

A morphism $\alpha: X \rightarrow Y$ between non-commutative schemes will be a right exact functor $\alpha^{*}: \operatorname{Qch}(Y) \rightarrow \operatorname{Qch}(X)$ possessing a right adjoint (denoted by $\alpha_{*}$ ). In this way the non-commutative schemes form a category (more accurately: a two-category).

In this paper we often view commutative schemes as non-commutative schemes. More precisely if $X$ is a commutative scheme, then $\mathrm{Qch}(X)$ will be the category of quasi-coherent sheaves on $X$. It is proved in [9] that this is a Grothendieck category. Furthermore $X$ can be recovered from $\mathrm{Qch}(X)[7,10,16]$.

If $X$ is a non-commutative scheme then we think of objects in $\mathrm{Qch}(X)$ as sheaves of right modules on $X$. To define the analogue of a sheaf of algebras on $X$ however we need a category of bimodules on $X$ (see [21] for the case where $X$ is commutative). The most obvious way to proceed is to define the category $\operatorname{Bimod}(X-Y)$ of $X-Y$-bimodules as the right exact functors $\mathrm{Q} \operatorname{ch}(X) \rightarrow \operatorname{Qch}(Y)$ commuting with direct limits. The action of a bimodule $\mathcal{N}$ on an object $\mathcal{M} \in \operatorname{Qch}(X)$ is written as $\mathcal{M} \otimes_{X} \mathcal{N}$.

If we define the "tensor product" of bimodules as composition then we can define algebra objects on $X$ as algebra objects in the category of $X-X$-bimodules and in this we may extend much of the ordinary commutative formalism. For example the identity functor $\mathrm{Q} \operatorname{ch}(X) \rightarrow \mathrm{Q} \operatorname{ch}(X)$ is a natural analogue of the structure sheaf, and as such it will be denoted by $o_{X}$. If $\mathcal{A}$ is an algebra object on $X$ then it is routine to define an abelian category $\operatorname{Mod}(\mathcal{A})$ of right- $\mathcal{A}$-modules. We have $\operatorname{Mod}\left(o_{X}\right)=\operatorname{Qch}(X)$. Unraveling all the definitions it turns out that $-\otimes_{X}-$ (the "tensor product" (composition) in the monoidal category $\operatorname{Bimod}(X-X)$ ) and $-\otimes_{o_{X}}-\left(\right.$ the tensor product over the algebra $\left.o_{X}\right)$ have the same meaning. We will use both notations, depending on the context.

Unfortunately $\operatorname{Bimod}(X-Y)$ appears not to be an abelian category and this represents a technical inconvenience which is solved in [22] by embedding $\operatorname{Bimod}(X-Y)$ into a larger category $\operatorname{BIMOD}(X-Y)$ consisting of "weak bimodules". The category $\operatorname{BIMOD}(X-Y)$ is opposite to the category of left exact functors $\mathrm{Qch}(Y) \rightarrow \mathrm{Qch}(X)$. Since left exact functors are determined by their values on injectives, they trivially form an abelian category. The category $\operatorname{Bimod}(X-Y)$ is the full category of 
$\operatorname{BIMOD}(X-Y)$ consisting of functors having a left adjoint. Or equivalently: functors commuting with direct products.

This being said, these technical complication will be invisible in this paper as all bimodules we encounter will be in $\operatorname{Bimod}(X-Y)$.

If $A$ be a graded algebra then the associated non-commutative scheme $X=$ $\operatorname{Proj} A$ is defined by $\operatorname{Qch}(X)=\operatorname{QGr}(A)=\operatorname{Gr}(A) / \operatorname{Tors}(A)$, as discussed above. Note that $\operatorname{Proj} A$ is only reasonably behaved when $A$ satisfies suitable homological conditions. See $[5,13]$. We denote the quotient functor $\operatorname{Gr}(A) \rightarrow \operatorname{QGr}(A)$ by $\pi$. The object $\pi A$ is denoted by $\mathcal{O}_{X}$. The "shift by $n$ " functor $\mathrm{Q} \operatorname{ch}(X)$ is written as $\mathcal{M} \mapsto \mathcal{M}(n)$ and the corresponding bimodule is written as $o_{X}(n)$. In particular $o_{X}=o_{X}(0)$ and $\mathcal{O}_{X}(n)=\mathcal{O} \otimes_{o_{X}} o_{X}(n)=\pi(A(n))$.

\section{Construction of the subalgebra $D$ of $\check{A}^{(2)}$}

We devote the rest of the paper to the proof of Theorem 1.1 as well as the construction of the non-commutative Cremona transform. The treatment of both constructions will be almost entirely parallel. So let $A$ be a 3 -dimensional Sklyanin algebra, which may be either quadratic or cubic, and put $X=\operatorname{Proj} A$.

As explained in the introduction (see also [3]) $A$ corresponds to a triple $(Y, \sigma, \mathcal{L})$, where $Y$ is smooth elliptic curve, $\sigma$ is a translation and $\mathcal{L}$ is a line bundle of degree $r$ on $Y$. The relation is given by the fact there is a regular central element $g \in A_{s+1}$ such that $A /(g)=B(Y, \sigma, \mathcal{L})$ where $B=B(Y, \sigma, \mathcal{L})$ is a so-called "twisted homogeneous coordinate ring" (see (1.1)).

Using the resulting equivalence of categories (see the introduction and [4])

$$
\operatorname{Proj} B \cong Y
$$

we will write $o_{Y}(n) \in \operatorname{Bimod}(Y-Y)$ for the shift by $n$-functor on Proj $B$. Then we have

$$
o_{Y}(1)=\sigma_{*}\left(-\otimes_{\mathcal{O}_{Y}} \mathcal{L}\right)
$$

(the tensor product takes place in the category of sheaves of $Y$-modules).

The inclusion functor $\mathrm{Qch}(Y) \subset \mathrm{Q} \operatorname{ch}(X)$ (i.e. the functor dual to the graded algebra morphism $A \rightarrow B$ ) has a left adjoint which we denote by $-\otimes_{o_{X}} o_{Y}$ (on the level of graded modules it corresponds to tensoring by $A / g A$ ). Note that in this way $o_{Y}$ is viewed as a $X-Y$-bimodule.

Below we will routinely regard a sheaf of $\mathcal{O}_{Y}$-modules $\mathcal{N}$ as an object in $\operatorname{Bimod}(Y-$ $Y$ ) by identifying it with the functor $-\otimes_{\mathcal{O}_{Y}} \mathcal{N}$. It is easy to see that the resulting functor

$$
\operatorname{Qch}(Y) \rightarrow \operatorname{Bimod}(Y-Y) \subset \operatorname{BIMOD}(Y-Y)
$$

is fully faithful and exact.

Similarly we regard an $Y-Y$-bimodule $\mathcal{M}$ as an $X-X$-bimodule by defining the corresponding functor to be

$$
\mathrm{Q} \operatorname{ch}(X) \stackrel{-\otimes_{o_{X} o_{Y}}}{\longrightarrow} \mathrm{Q} \operatorname{ch}(Y) \stackrel{-\otimes_{o_{Y}} \mathcal{M}}{\longrightarrow} \mathrm{Q} \operatorname{ch}(Y) \hookrightarrow \mathrm{Q} \operatorname{ch}(X)
$$

In this way $o_{Y}$ becomes an $X-X$-bimodule and one checks that it is in fact an algebra quotient of $o_{X}$. Note that $o_{Y}$ now denotes both an algebra on $X$ and an algebra on $Y$ (the identity functor) but for both interpretations we have $\operatorname{Mod}\left(o_{Y}\right) \cong$ $\operatorname{Qch}(Y)$. 
For use in the sequel we write

$$
o_{X}(-Y)=\operatorname{ker}\left(o_{X} \rightarrow o_{X}\right)
$$

$o_{X}(-Y)$ is the ideal in $o_{X}$ corresponding to the graded ideal $g A \subset A$. Note that since $g$ is central we have in fact $o_{X}(-Y)=o_{X}(-3)$.

If $\mathcal{M} \in \mathrm{Q} \operatorname{ch}(X)$ then we define the "global sections" of $\mathcal{M}$ as

$$
\Gamma(X, \mathcal{M})=\operatorname{Hom}_{X}\left(\mathcal{O}_{X}, \mathcal{M}\right)
$$

Similarly we define the global sections of an $X-X$-bimodule $\mathcal{N}$ as in $[22$, Section $3.5]$ :

$$
\Gamma(X, \mathcal{N}):=\operatorname{Hom}\left(\mathcal{O}_{X}, \mathcal{O}_{X} \otimes_{o_{X}} \mathcal{N}\right)
$$

Use of the functor $\Gamma(X,-)$ on bimodules requires some care since it is apriori not left exact. However in our applications it will be.

Note that $\mathcal{N}$ is an algebra object in the category of bimodules then $\Gamma(X, \mathcal{N})$ is in fact an algebra for purely formal reasons. The same holds true for graded algebras and $\mathbb{Z}$-algebras.

It is easy to see that $A_{n}$ is equal to the global sections of $o_{X}(n)$ :

$$
\begin{aligned}
\Gamma\left(X, o_{X}(n)\right) & :=\operatorname{Hom}_{X}\left(\mathcal{O}_{X}, \mathcal{O}_{X}(n)\right) \\
& =\operatorname{Hom}_{\mathrm{QGr}(A)}(\pi(A), \pi(A(n))) \\
& =\operatorname{Hom}_{\operatorname{Gr}(A)}(A, A(n)) \quad[5, \text { Theorem 8.1(5)] } \\
& =A_{n}
\end{aligned}
$$

where the third equality follows from the AS-regularity of $A$. Thus for the $\mathbb{Z}$-algebra associated to the two-Veronese of $A$ we have:

$$
\check{A}_{m, n}^{(2)}=\Gamma\left(X, o_{X}(2(n-m))\right)=\Gamma\left(X, o_{X}(-2 m) \otimes_{o_{X}} o_{X}(2 n)\right)
$$

Below $\left(p_{i}\right)_{i}$ is a collection of points on $Y$ : three distinct points in case $(r, s)=$ $(3,2)$ and one point in case $(r, s)=(2,3)$. Let $d=\sum_{i} p_{i}$ be the corresponding divisor on $Y$. As above we consider $\mathcal{O}_{d}$ as a $Y-Y$-bimodule but to avoid confusion we write it as $o_{d}$. Following our convention above we also consider $o_{d}$ as an $X$ bimodule. Put

$$
\begin{aligned}
m_{d, Y} & =\operatorname{ker}\left(o_{Y} \rightarrow o_{d}\right) \\
m_{d} & =\operatorname{ker}\left(o_{X} \rightarrow o_{d}\right)
\end{aligned}
$$

Clearly $m_{d, Y} \in \operatorname{Bimod}(Y-Y)$ as $m_{d, Y}$ corresponds to an ordinary ideal sheaf in $\mathcal{O}_{Y}$ (see (4.1) above). The fact that $m_{d} \in \operatorname{Bimod}(X-X)$ follows by applying $[22$, Corollary 5.5.6] repeatedly for the different $p_{i}$.

Finally consider the following bimodules over $X$, respectively $Y$ :

$$
\begin{aligned}
\left(\mathcal{D}_{Y}\right)_{m, n} & = \begin{cases}o_{Y}(-2 m) \otimes_{o_{Y}} m_{\tau^{-m} d, Y} \ldots m_{\tau^{-n+1} d, Y} \otimes_{o_{Y}} o_{Y}(2 n) & \text { if } n \geqslant m \\
0 & \text { if } n<m\end{cases} \\
\mathcal{D}_{m, n} & = \begin{cases}o_{X}(-2 m) \otimes_{o_{X}} m_{\tau^{-m} d} \ldots m_{\tau^{-n+1} d} \otimes_{o_{X}} o_{X}(2 n) & \text { if } n \geqslant m \\
0 & \text { if } n<m\end{cases}
\end{aligned}
$$

where $\tau=\sigma^{s+1}$. Here $m_{\tau^{-k} d} \ldots m_{\tau^{-l} d}$ is the image of

$$
m_{\tau^{-l} d} \otimes_{X} \cdots \otimes_{X} m_{\tau^{-l} d} \longrightarrow o_{X} \otimes_{X} \cdots \otimes_{X} o_{X}=o_{X}
$$

A priori this image lies only in $\operatorname{BIMOD}(X-X)$ but with the same method as the proof of [22, Proposition 6.1.1] one verifies that it lies in fact in $\operatorname{Bimod}(X-X)$. 
The collections of bimodules $\mathcal{D} \stackrel{\text { def }}{=} \bigoplus_{m, n} \mathcal{D}_{m, n}, \mathcal{D}_{Y} \stackrel{\text { def }}{=} \bigoplus_{m, n}\left(\mathcal{D}_{Y}\right)_{m, n}$ represent $\mathbb{Z}$-algebra objects respectively in $\operatorname{Bimod}(X-X)$ and $\operatorname{Bimod}(Y-Y)$. For example the product

is given by

$$
\mathcal{D}_{m, n} \otimes_{o_{X}} \mathcal{D}_{n, p}
$$

$$
\begin{gathered}
o_{X}(-2 m) \otimes_{o_{X}} m_{\tau^{-m}} \ldots m_{\tau^{-n+1} d} \otimes_{o_{X}} o_{X}(2 n) \otimes_{o_{X}} o_{X}(-2 n) \otimes_{o_{X}} m_{\tau^{-n} d} \ldots m_{\tau^{-p+1} d} \otimes_{o_{X}} o_{X}(2 p) \rightarrow \\
o_{X}(-2 m) \otimes_{o_{X}} m_{\tau^{-m} d} \ldots m_{\tau^{-n+1} d} \otimes_{o_{X}} m_{\tau^{-n}} \ldots m_{\tau^{-p+1} d} \otimes_{o_{X}} o_{X}(2 p) \rightarrow \\
o_{X}(-2 m) \otimes_{o_{X}} m_{\tau^{-m} d} \ldots m_{\tau^{-n+1} d} m_{\tau^{-n} d} \ldots m_{\tau^{-p+1} d} \otimes_{o_{X}} o_{X}(2 p)
\end{gathered}
$$

Denote the global sections of $\mathcal{D}$ and $\mathcal{D}_{Y}$ by $D, D_{Y}$ respectively. Thus $D$ and $D_{Y}$ are both $\mathbb{Z}$-algebras.

The inclusion $\mathcal{D}_{m, n} \hookrightarrow o_{X}(2(n-m))$ gives rise to an inclusion of $\mathbb{Z}$-algebras $D \hookrightarrow \check{A}^{(2)}$ by using [22, Lemma 8.2.1] with $\mathcal{E}=\mathcal{O}_{X}$. This is the sought sub- $\mathbb{Z}$ algebra of $\check{A}^{(2)}$.

\section{Analysis of $D_{Y}$}

Our aim is to show that $D$ is a quadratic AS-regular $\mathbb{Z}$-algebra. The first step in understanding $D$ is showing that the quotient $\mathbb{Z}$-algebra $D_{Y}$ is a $\mathbb{Z}$-analogue of a twisted homogeneous coordinate ring (see $\S 2$ ). We do this next.

We have to find an elliptic helix $\left\{\mathcal{L}_{i}\right\}_{i}$ as defined in $\S 2$ such that

$$
\left(D_{Y}\right)_{m, n}=B\left(Y,\left\{\mathcal{L}_{i}\right\}_{i}\right)_{m, n}:=\Gamma\left(Y, \mathcal{L}_{m} \otimes \ldots \otimes \mathcal{L}_{n-1}\right)
$$

The functor $-\otimes_{o_{Y}} m_{d, Y}$ is given by $-\otimes_{\mathcal{O}_{Y}} \mathcal{M}_{d, Y}$ where $\mathcal{M}_{d, Y}$ is the ideal sheaf of $d$ on $Y$ (see (4.1) above). Moreover as we have already mentioned $o_{Y}(1)=\sigma_{*}\left(-\otimes_{k} \mathcal{L}\right)$ (using the notations of [21] we could write this as: $m_{d, Y}={ }_{1}\left(\mathcal{M}_{d, Y}\right)_{1}$ and $o_{Y}(1)=$ $\left.{ }_{1} \mathcal{L}_{\sigma}\right)$. Using the fact that $-\otimes o_{Y}(2 n)$ is an autoequivalence we compute for $n \geqslant m$

$$
\begin{aligned}
& \left(D_{Y}\right)_{m, n}=\operatorname{Hom}\left(\mathcal{O}_{Y}, \mathcal{O}_{Y}(-2 m) \otimes m_{\tau^{-m} d, Y} \ldots m_{\tau^{-n+1} d, Y} \otimes o_{Y}(2 n)\right) \\
& =\operatorname{Hom}\left(\mathcal{O}_{Y}(-2 n), \mathcal{O}_{Y}(-2 m) \otimes m_{\tau^{-m} d, Y} \ldots m_{\tau^{-n+1} d, Y}\right) \\
& =\operatorname{Hom}\left(\left(\mathcal{L} \otimes \ldots \otimes \mathcal{L}^{\sigma^{2 n-1}}\right)^{-1},\left(\mathcal{L} \otimes \ldots \otimes \mathcal{L}^{\sigma^{2 m-1}}\right)^{-1} \otimes \mathcal{M}_{\tau^{-m} d, Y} \ldots \mathcal{M}_{\tau^{-n+1} d, Y}\right) \\
& =\operatorname{Hom}\left(\mathcal{O}_{Y}, \mathcal{M}_{\tau^{-m} d, Y} \ldots \mathcal{M}_{\tau^{-n+1} d, Y} \otimes \mathcal{L}^{\sigma^{2 m}} \otimes \ldots \otimes \mathcal{L}^{\sigma^{2 n-1}}\right) \\
& =\Gamma\left(Y, \mathcal{M}_{\tau^{-m} d, Y} \ldots \mathcal{M}_{\tau^{-n+1} d, Y} \otimes \mathcal{L}^{\sigma^{2 m}} \otimes \ldots \otimes \mathcal{L}^{\sigma^{2 n-1}}\right) \\
& =\Gamma\left(Y, \mathcal{L}_{m} \otimes \ldots \otimes \mathcal{L}_{n-1}\right)
\end{aligned}
$$

with

$$
\mathcal{L}_{i}=\mathcal{M}_{\tau^{-i} d, Y} \otimes \mathcal{L}^{\sigma^{2 i}} \otimes \mathcal{L}^{\sigma^{2 i+1}}
$$

A routine but somewhat tedious verification shows that the isomorphism constructed in (5.1) sends the product on the left to the obvious product on the right corresponding to the tensorproduct.

We now have to check that the $\left(\mathcal{L}_{i}\right)_{i}$ constitute an elliptic helix as introduced in $\S 2$. Using our standing hypothesis that $Y$ is smooth (since $A$ was assumed to be a Sklyanin algebra) we must verify the following facts 
(1) $\operatorname{deg} \mathcal{L}_{i}=3$

(2) $\mathcal{L}_{0} \not \mathcal{L}_{1}$.

(3) $\mathcal{L}_{i} \otimes \mathcal{L}_{i+1}^{-2} \otimes \mathcal{L}_{i+2} \cong \mathcal{O}_{Y}$.

We use the following lemma.

Lemma 5.1. If $A$ is quadratic, one has

$$
\sigma^{*}\left(\mathcal{L}_{i}\right)=\mathcal{L}_{i+1}
$$

and if $A$ is cubic

$$
\psi^{*}\left(\mathcal{L}_{i}\right)=\mathcal{L}_{i+1}
$$

where $\psi$ is an arbitrary translation satisfying $\psi^{3}=\sigma^{4}$.

Proof. We compute in the quadratic case

$$
\begin{aligned}
\sigma^{*}\left(\mathcal{L}_{i}\right) \otimes \mathcal{L}_{i+1}^{-1} & =\mathcal{M}_{\tau^{-i} d, Y}^{\sigma} \otimes \mathcal{L}^{\sigma^{2 i+1}} \otimes \mathcal{L}^{\sigma^{2 i+2}} \otimes \mathcal{M}_{\tau^{-i-1} d, Y}^{-1} \otimes\left(\mathcal{L}^{\sigma^{2 i+2}}\right)^{-1} \otimes\left(\mathcal{L}^{\sigma^{2 i+3}}\right)^{-1} \\
& =\mathcal{M}_{\tau^{-i} d, Y}^{\sigma} \otimes \mathcal{L}^{\sigma^{2 i+1}} \otimes\left(\mathcal{M}_{\tau^{-i} d, Y}^{\sigma^{3}}\right)^{-1} \otimes\left(\mathcal{L}^{\sigma^{2 i+3}}\right)^{-1}
\end{aligned}
$$

Since $\sigma$ is a translation there is an invertible sheaf $\mathcal{N}$ of degree zero on $Y$ such that for each invertible sheaf $\mathcal{M}$ on $Y$ we have the following identities in $\operatorname{Pic}(Y)$ :

$$
\left[\sigma^{*} \mathcal{M}\right]=[\mathcal{M}]+\operatorname{deg}(\mathcal{M}) \cdot[\mathcal{N}]
$$

(This statement is true in even higher generality, see [23, Theorem 4.2.3]) Thus

$$
\left[\sigma^{*}\left(\mathcal{L}_{i}\right) \otimes \mathcal{L}_{i+1}^{-1}\right]=\left(\operatorname{deg}\left(\mathcal{M}_{\tau^{-i} d, Y}\right)+\operatorname{deg}\left(\mathcal{L}^{\sigma^{2 i}}\right)-3 \operatorname{deg}\left(\mathcal{M}_{\tau^{-i} d, Y}\right)-3 \operatorname{deg}\left(\mathcal{L}^{\sigma^{2 i}}\right)\right)[\mathcal{N}]=0
$$

taking into account that in the quadratic case

$$
\begin{aligned}
\operatorname{deg}\left(\mathcal{M}_{\tau^{-i} d, Y}\right) & =-\operatorname{deg} d=-3 \\
\operatorname{deg}\left(\sigma^{*^{2 i}} \mathcal{L}\right) & =3
\end{aligned}
$$

Now we consider the cubic case. It will be convenient to introduce a translation $\sigma_{3}$ which is a cube root of $\sigma$

$$
\left.\sigma_{3}^{4^{*}}\left(\mathcal{L}_{i}\right) \otimes \mathcal{L}_{i+1}^{-1}=\mathcal{M}_{\tau^{-i} d, Y}^{\sigma_{3}^{4}} \otimes \mathcal{L}^{\sigma_{3}^{6 i+4}} \otimes \mathcal{L}^{\sigma_{3}^{6 i+7}} \otimes\left(\mathcal{M}_{\tau^{-i} d, Y}^{\sigma_{3}^{12}}\right)^{-1} \otimes\left(\mathcal{L}^{\sigma_{3}^{6 i+6}}\right)^{-1} \otimes \mathcal{L}^{\sigma_{3}^{6 i+9}}\right)^{-1}
$$

Let $\mathcal{N}_{3}$ be a line bundle of degree zero such that for any line bundle $\mathcal{M}$

$$
\left[\sigma_{3}^{*} \mathcal{M}\right]=[\mathcal{M}]+\operatorname{deg}(\mathcal{M}) \cdot\left[\mathcal{N}_{3}\right]
$$

We obtain

$$
\begin{gathered}
{\left[\sigma_{3}^{4^{*}}\left(\mathcal{L}_{i}\right) \otimes \mathcal{L}_{i+1}^{-1}\right]=\left(4 \operatorname{deg}\left(\mathcal{M}_{\tau^{-i} d, Y}\right)+4 \operatorname{deg}\left(\mathcal{L}^{\sigma_{3}^{6 i}}\right)+7 \operatorname{deg}\left(\mathcal{L}^{\sigma_{3}^{6 i}}\right)-12 \operatorname{deg}\left(\mathcal{M}_{\tau^{-i} d, Y}\right)\right.} \\
\left.-6 \operatorname{deg}\left(\mathcal{L}^{\sigma_{3}^{6 i}}\right)-9 \operatorname{deg}\left(\mathcal{L}^{\sigma_{3}^{6 i}}\right)\right)\left[\mathcal{N}_{3}\right]=0
\end{gathered}
$$

taking into account that this time

$$
\begin{aligned}
\operatorname{deg}\left(\mathcal{M}_{\tau^{-i} d, Y}\right) & =-\operatorname{deg} d=-1 \\
\operatorname{deg}\left(\mathcal{L}^{\sigma_{3}^{6 i}}\right) & =2
\end{aligned}
$$

We now verify that $\left(\mathcal{L}_{i}\right)_{i}$ is an elliptic helix. Condition (1) is immediate and condition (3) follows from Lemma 5.1. Assume that (2) is false in the quadratic case. Then $\sigma^{*}\left(\mathcal{L}_{0}\right)=\mathcal{L}_{0}$. In other words $\sigma$ is translation by a point of order three. But this contradicts our assumption that $A$ is a Sklyanin algebra. Now assume that (2) is false in the cubic case. The $\psi$ is a translation by a point of order three and 
from the definition of $\psi$ it follows that $\sigma$ is translation by a point of order four, again contradicting the fact that $A$ is Sklyanin algebra.

\section{Showing that $D$ is AS-Regular}

For use below recall some some commutation formulas. First note that since $o_{Y}(1)=\sigma_{*}\left(-\otimes_{\mathcal{O}_{Y}} \mathcal{L}\right)$ we have

$$
o_{d} \otimes_{o_{Y}} o_{Y}(1)=o_{Y}(1) \otimes_{o_{Y}} o_{\sigma d}
$$

(we may see this by applying both sides to an object in $\mathrm{Qch}(Y)$ ). Using the definitions of $m_{d}, m_{d, Y}$ (see (4.2), (4.3)) we deduce from this

$$
\begin{gathered}
m_{d, Y} \otimes_{o_{Y}} o_{Y}(1)=o_{Y}(1) \otimes_{o_{Y}} m_{\sigma d, Y} \\
m_{d} \otimes_{o_{X}} o_{X}(1)=o_{X}(1) \otimes_{o_{X}} m_{\sigma d}
\end{gathered}
$$

Similar formulas also hold for longer products of $m$ 's such as for example appear in the definition of $\left(\mathcal{D}_{Y}\right)_{m, n}$ and $\mathcal{D}_{m, n}$.

If $\mathcal{M}$ is a bimodule then we will write $(a) \mathcal{M}$ for $\mathcal{O}_{X}(a) \otimes_{o_{X}} \mathcal{M}$. Thus the "right structure" of $\mathcal{M}$ is $(0) \mathcal{M}$. For the sequel we need a resolution of $(a) \mathcal{D}_{m, m+1}$. In the quadratic case we use the following lemma.

Lemma 6.1. Let $A$ be a quadratic AS-regular algebra of dimension 3. Let $q_{1}, q_{2}, q_{3}$ be distinct non-collinear points in $Y$ and let $Q_{1}, Q_{2}, Q_{3}$ be the corresponding point modules. ${ }^{1}$. Pick an $m$ in $\left(Q_{1} \oplus Q_{2} \oplus Q_{3}\right)_{0}$ whose three components are non-zero and let $M=m A$. Then the minimal resolution of $M$ has the following form

$$
0 \rightarrow A(-3)^{\oplus 2} \rightarrow A(-2)^{\oplus 3} \rightarrow A \rightarrow M \rightarrow 0
$$

Proof. Let $g$ be the normalizing element of degree three in $A$ and let $B=A / g A$. By using the explicit category equivalence $\mathrm{Q} \operatorname{ch}(B) \cong \mathrm{Q} \operatorname{Gr}(Y)[4]$ one easily proves that the map $B_{\geqslant 1} \rightarrow M_{\geqslant 1}$ is surjective. Whence the corresponding map $u: A_{\geqslant 1} \rightarrow M_{\geqslant 1}$ is also surjective.

Look at the exact sequence

$$
0 \rightarrow \operatorname{ker} u \rightarrow A_{\geqslant 1} \rightarrow M_{\geqslant 1} \rightarrow 0
$$

Tensoring this exact sequence with $k$ yields an exact sequence

$$
\operatorname{Tor}_{1}^{A}\left(M_{\geqslant 1}, k\right) \rightarrow \operatorname{ker} u \otimes_{A} k \rightarrow A_{\geqslant 1} \otimes_{A} k \stackrel{\bar{u}}{\rightarrow} M_{\geqslant 1} \otimes k \rightarrow 0
$$

Now both $A_{\geqslant 1}$ and $M_{\geqslant 1}$ are generated in degree one and furthermore $\operatorname{dim} A_{1}=$ $\operatorname{dim} M_{1}$. Hence it follows that $\bar{u}$ is an isomorphism. Therefore $\operatorname{ker} u \otimes_{A} k$ is a quotient of $\operatorname{Tor}_{1}^{A}\left(M_{\geqslant 1}, k\right)$. From the fact that $M_{\geqslant 1}$ is a sum of shifted point modules we compute that $\operatorname{Tor}_{1}^{A}\left(M_{\geqslant 1}, k\right)=k(-2)^{3}$. Thus ker $u$ is a quotient of $A(-2)^{3}$. Now using the fact that $M$ has no torsion and hence has projective dimension two we may now complete the full resolution of $M$ using a Hilbert series argument.

Note that

$$
\mathcal{D}_{m, m+1}=o_{X}(-2 m) \otimes_{o_{X}} m_{\tau^{-m} d} \otimes_{o_{X}} o_{X}(2(m+1))
$$

and thus

$$
\mathcal{O}_{X}(a) \otimes_{o_{X}} \mathcal{D}_{m, m+1}=\left(\mathcal{O}_{X} \otimes_{o_{X}} m_{\sigma^{2 m-a} \tau^{-m} d}\right)(a+2)
$$

\footnotetext{
${ }^{1} \mathrm{~A}$ point module over $A$ is a graded right $A$-module generated in degree zero with Hilbert function $1,1,1,1,1, \ldots$. There is a $1-1$ correspondence between points in $Y$ and point modules over $A$. See $[2]$.
} 
NONCOMMUTATIVE VERSIONS OF CLASSICAL BIRATIONAL TRANSFORMATIONS. 11

where

$$
\mathcal{O}_{X} \otimes_{o_{X}} m_{\sigma^{2 m-a} \tau^{-m} d}=\operatorname{ker}\left(\mathcal{O}_{X} \rightarrow \mathcal{O}_{\sigma^{2 m-a} \tau^{-m} d}\right)
$$

Thus $\mathcal{O}_{X} \otimes_{o_{X}} m_{\sigma^{2 m-a} \tau^{-m}}$ is of the form $\pi(\operatorname{ker}(A \rightarrow M))$ with $M$ as in Lemma 6.1. We conclude that we have a resolution of $(a) \mathcal{D}_{m, m+1}$ of the form

$$
0 \rightarrow \mathcal{O}_{X}(a-1)^{\oplus 2} \rightarrow \mathcal{O}_{X}(a)^{\oplus 3} \rightarrow(a) \mathcal{D}_{m, m+1} \rightarrow 0
$$

This resolution is actually of the form

$$
0 \rightarrow \mathcal{O}_{X}(a-1)^{\oplus 2} \rightarrow \mathcal{O}_{X}(a) \otimes_{k} D_{m, m+1} \rightarrow(a) \mathcal{D}_{m, m+1} \rightarrow 0
$$

In the cubic case the resolution will follow from the next lemma:

Lemma 6.2. Let $A$ be a cubic AS-regular algebra of dimension 3. Let $p$ be a point in $Y$ and let $P$ be the corresponding point module. Then there is a complex of the following form:

$$
0 \rightarrow A(-5) \stackrel{(\zeta, 0)}{\longrightarrow} A(-4)^{\oplus 2} \oplus A(-3) \rightarrow A(-2)^{\oplus 3} \rightarrow A \rightarrow P \rightarrow 0
$$

where $\zeta$ is part of the minimal resolution of $k$ as given in [1, Theorem 1.5.]

$$
0 \rightarrow A(-4) \stackrel{\zeta}{\rightarrow} A(-3)^{2} \stackrel{\varepsilon}{\rightarrow} A(-1)^{2} \stackrel{\delta_{0}}{\rightarrow} A \stackrel{\gamma}{\rightarrow} k \rightarrow 0
$$

Moreover the complex (6.3) is exact everywhere except at $A$ where it has onedimensional cohomology, concentrated in degree one.

Proof. From [2, Proposition 6.7.] we know $P$ has the following (minimal) resolution:

$$
0 \rightarrow A(-3) \rightarrow A(-2) \oplus A(-1) \rightarrow A \rightarrow P \rightarrow 0
$$

Combining this with the minimal resolution for $k$ we get the following diagram

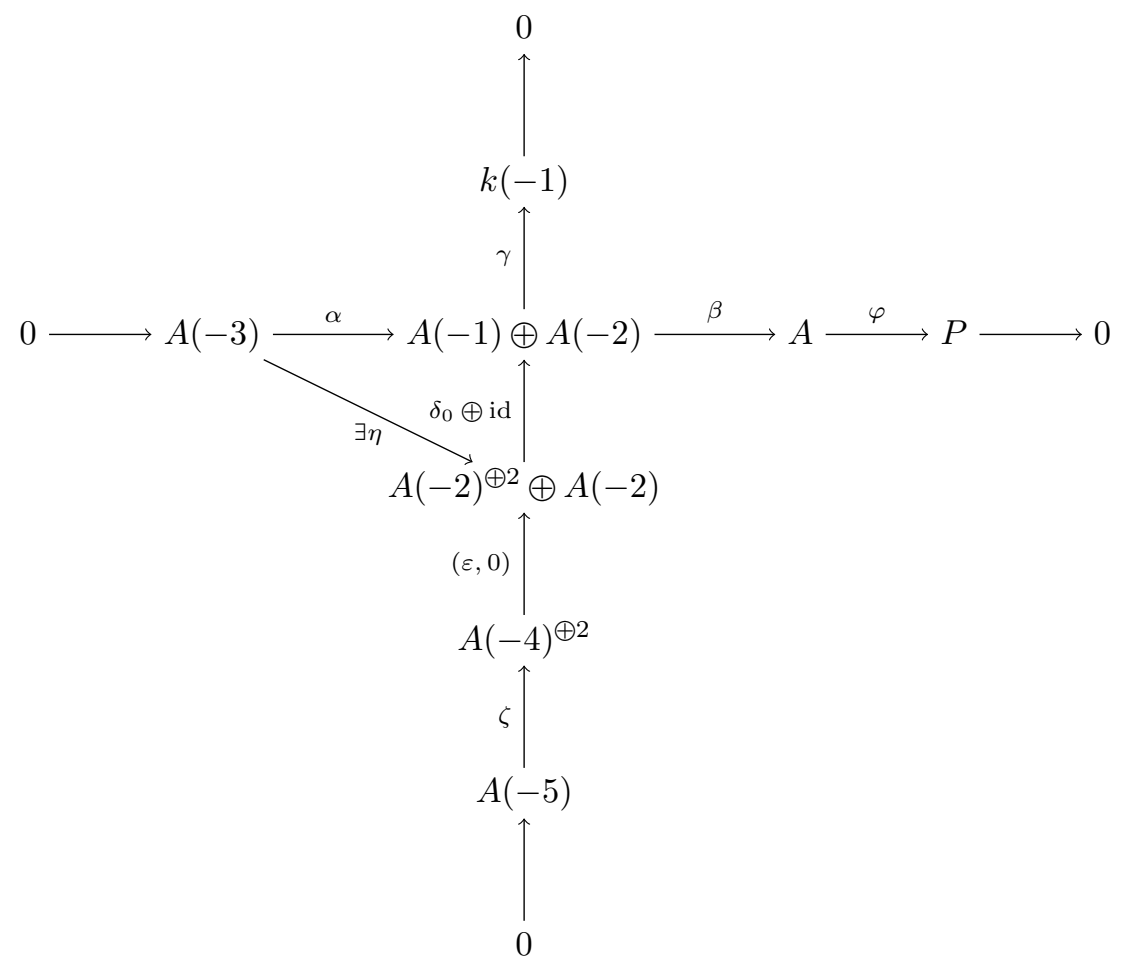


Put $\delta=\delta_{0} \oplus$ id. The existence of the map $\eta$ such that $\delta \circ \eta=\alpha$ follows from the projectivity of $A(-3)$ and the fact that $\gamma \circ \alpha$ is zero by degree reasons. By diagram chasing one easily finds that $\operatorname{ker}(\beta \circ \delta)=\operatorname{im}(\varepsilon) \oplus \operatorname{im}(\eta)$ and hence we end up with the following complex:

$$
0 \rightarrow A(-5) \stackrel{\zeta \oplus 0}{\longrightarrow} A(-4)^{\oplus 2} \oplus A(-3) \stackrel{\eta \oplus \varepsilon}{\longrightarrow} A(-2)^{\oplus 3} \stackrel{\beta \circ \delta}{\longrightarrow} A \stackrel{\varphi}{\longrightarrow} P \rightarrow 0
$$

Using diagram chasing again one easily checks that this complex is exact everywhere except at $A$. We then conclude with a Hilbert series argument.

In a similar way as in the quadratic case we conclude that $(a) \mathcal{D}_{m, m+1}$ has a resolution of the form

$$
0 \rightarrow \mathcal{O}_{X}(a-3) \stackrel{(\zeta, 0)}{\longrightarrow} \mathcal{O}_{X}(a-2)^{\oplus 2} \oplus \mathcal{O}_{X}(a-1) \rightarrow \mathcal{O}_{X}(a)^{\oplus 3} \rightarrow(a) \mathcal{D}_{m, m+1} \rightarrow 0
$$

which is actually of the form

$$
0 \rightarrow \mathcal{J}(a) \oplus \mathcal{O}_{X}(a-1) \rightarrow \mathcal{O}_{X}(a) \otimes_{k} D_{m, m+1} \rightarrow(a) \mathcal{D}_{m, m+1} \rightarrow 0
$$

where

$$
\mathcal{J} \stackrel{\text { def }}{=} \operatorname{coker}\left(\mathcal{O}_{X}(-3) \stackrel{\zeta}{\rightarrow} \mathcal{O}_{X}(-2)^{\oplus 2}\right)
$$

We will now prove some vanishing results. An object in $\mathrm{Q} \operatorname{ch}(X)$ will be said to have finite length if it is a finite extension of objects of the form $\mathcal{O}_{p}, p \in Y$. Likewise an object in $\operatorname{Bimod}(X-X)$ will be said to have finite length if it is a finite extension of $o_{p}$ for $p \in Y$. The objects of finite length are fully understood, see [22, Chapter 5]. Note that by [22, Proposition 5.5.2] $o_{p}$ is a simple object in $\operatorname{Bimod}(X-X)$ so the Jordan-Holder theorem applies to finite length bimodules.

Lemma 6.3. A finite length object in $\mathrm{Q} \operatorname{ch}(X)$ has no higher cohomology.

Proof. For an object of the form $o_{p}$ this follows from [22, Proposition 5.1.2] with $\mathcal{F}=\mathcal{O}_{X}$. The general case follows from the long exact sequence for Ext.

Lemma 6.4. $H^{2}\left(X,(-l) \mathcal{D}_{m, n}\right)=0$ for $l \leqslant 2 n-2 m+s$.

Proof. We only need to consider the case $n \geqslant m$. This follows from the fact that $(-l) \mathcal{D}_{m, n} \subset \mathcal{O}_{X}(2 n-2 m-l)$ with finite length cokernel and from the standard vanishing properties on $\mathrm{Q} \operatorname{Gr}(A)$ (see for example [5, Theorem 8.1]).

Lemma 6.5. $H^{1}\left(X,(a) \mathcal{D}_{m, n}\right)=0$ for $a \geqslant-s+1$.

Proof. We only need to consider the case $n \geqslant m$. The proof for $a \geqslant-1$ is similar in the cases $(r, s)=(2,3)$ and $(r, s)=(3,2)$ so we will give the proof for the first case as it is slightly longer. Afterwards we will consider the case $(r, s)=(2,3)$ and $a=-2$.

Suppose $(r, s)=(2,3)$ and $a \geqslant-1$. We prove $H^{1}\left(X,(a) \mathcal{D}_{m, n}\right)=0$ by induction on $n-m$. As $(a) \mathcal{D}_{m, m}=\mathcal{O}_{X}(a)$ the base case follows from the standard vanishing on $X$.

For the induction step we proceed as follows: From [22, Theorem 5.5.10] and the fact that $\mathcal{D}_{m, n} \subset o_{X}(2 n-2 m)$ with finite length cokernel we may deduce that the kernel of the obvious surjective map

$$
\mathcal{D}_{m, m+1} \otimes_{X} \mathcal{D}_{m+1, n} \rightarrow \mathcal{D}_{m, n}
$$


has finite length. Using [22, Lemma 8.2.1] we see that this remains the case if we left tensor with $\mathcal{O}_{X}(a)$. Thus we obtain a short exact sequence in $\mathrm{Q} \operatorname{ch}(X)$

$$
0 \rightarrow \text { f.l. } \rightarrow(a) \mathcal{D}_{m, m+1} \otimes_{X} \mathcal{D}_{m+1, n} \rightarrow(a) \mathcal{D}_{m, n} \rightarrow 0
$$

from which we find $H^{1}\left(X,(a) \mathcal{D}_{m, n}\right)=H^{1}\left(X,(a)\left(\mathcal{D}_{m, m+1} \otimes_{X} \mathcal{D}_{m+1, n}\right)\right)$ by Lemma 6.3. From (6.5) we obtain an exact sequence

$$
\begin{aligned}
\operatorname{Tor}_{1}^{o_{X}}\left((a) \mathcal{D}_{m, m+1}, \mathcal{D}_{m+1, n}\right) \rightarrow \mathcal{J}(a) \otimes_{X} \mathcal{D}_{m+1, n} \oplus(a-1) \mathcal{D}_{m+1, n} & \rightarrow \\
D_{m . m+1} \otimes_{k}(a) \mathcal{D}_{m+1, n} \rightarrow(a) \mathcal{D}_{m, m+1} \otimes_{X} \mathcal{D}_{m+1, n} & \rightarrow 0
\end{aligned}
$$

One deduces again, for example using [22, Theorem 5.5.10], that $\operatorname{Tor}_{1}^{o X}\left((a) \mathcal{D}_{m, m+1}, \mathcal{D}_{m+1, n}\right)$ has finite length. It is clear that $(a-1) \mathcal{D}_{m+1, n}$ has no finite length subobjects. We claim this is the same for $\mathcal{J}(a) \otimes_{X} \mathcal{D}_{m+1, n}$. Indeed tensoring the short exact sequence

$$
0 \rightarrow \mathcal{J}(a) \rightarrow \mathcal{O}_{X}(a)^{\oplus 2} \rightarrow \mathcal{O}_{X}(a+1) \rightarrow 0
$$

on the right with $\mathcal{D}_{m+1, n}$ and using Tor-vanishing [22, Theorem 8.2.1] we obtain a short exact sequence

$$
0 \rightarrow \mathcal{J}(a) \otimes_{X} \mathcal{D}_{m+1, n} \rightarrow(a) \mathcal{D}_{m+1, n}^{\oplus 2} \rightarrow(a+1) \mathcal{D}_{m+1, n} \rightarrow 0
$$

Hence in particular $\mathcal{J}(a) \otimes_{X} \mathcal{D}_{m+1, n} \subset(a) \mathcal{D}_{m+1, n}^{\oplus 2}$ is torsion free. We conclude that (6.7) becomes in fact a short exact sequence

$0 \rightarrow \mathcal{J}(a) \otimes_{X} \mathcal{D}_{m+1, n} \oplus(a-1) \mathcal{D}_{m+1, n} \rightarrow D_{m, m+1} \otimes_{k}(a) \mathcal{D}_{m+1, n} \rightarrow(a) \mathcal{D}_{m, m+1} \otimes_{X} \mathcal{D}_{m+1, n} \rightarrow 0$

We find that $H^{1}\left(X,(a)\left(\mathcal{D}_{m, m+1} \otimes_{X} \mathcal{D}_{m+1, n}\right)\right)$ is sandwiched between a direct sum of copies of $H^{1}\left(X,(a) \mathcal{D}_{m+1, n}\right)$ (=0 by the induction hypothesis) and a direct sum of copies of $H^{2}\left(X, \mathcal{J}(a) \otimes_{X} \mathcal{D}_{m+1, n}\right)$. Now $H^{2}\left(X, \mathcal{J}(a) \otimes_{X} \mathcal{D}_{m+1, n}\right)$ is trivial as well because it is sandwiched between a direct sum of copies of $H^{2}\left((a-2) \mathcal{D}_{m+1, n}\right)$ (=0 by Lemma 6.4) and $H^{3}\left(X,(a-3) \mathcal{D}_{m+1, n}\right)\left(=0\right.$ as $\left.H^{3}(X,-)=0\right)$.

We now prove $H^{1}\left(X,(-2) \mathcal{D}_{m, n}\right)=0$ when $(r, s)=(2,3)$. This can also be done by induction on $n-m$. The case $n=m$ again follows from the standard vanishing on $X$. For the induction step recall that for any point $q$ there is an exact sequence:

$$
0 \rightarrow \mathcal{O}_{X}(-3) \rightarrow \mathcal{O}_{X}(-2) \oplus \mathcal{O}_{X}(-1) \rightarrow \mathcal{O}_{X} \otimes_{X} m_{q} \rightarrow 0
$$

Applying $-\otimes_{X} \mathcal{D}_{m+1, n}$ yields an exact sequence

$$
0 \rightarrow(-3) \mathcal{D}_{m+1, n} \rightarrow(-2) \mathcal{D}_{m+1, n} \oplus(-1) \mathcal{D}_{m+1, n} \rightarrow \mathcal{O}_{X} \otimes_{X} m_{q} \otimes_{X} \mathcal{D}_{m+1, n} \rightarrow 0
$$

where the injectivity of $(-3) \mathcal{D}_{m+1, n} \rightarrow(-2) \mathcal{D}_{m+1, n} \oplus(-1) \mathcal{D}_{m+1, n}$ is a torsion/torsion free argument as above in the derivation of (6.9). In particular we can consider a long exact sequence of cohomology groups. As in this sequence $H^{1}\left(X,(-2) \mathcal{D}_{m, n}\right)=$ $H^{1}\left(X, m_{\tau^{-m-1} p} \otimes_{X} \mathcal{D}_{m+1, n}\right)$ is sandwiched between $H^{1}\left(X,(-2) \mathcal{D}_{m+1, n}\right) \oplus H^{1}\left(X,(-1) \mathcal{D}_{m+1, n}\right)$ and $H^{2}\left(X,(-3) \mathcal{D}_{m+1, n}\right)$ we can conclude by the induction hypothesis, the case $a \geqslant-1$ which was already done and Lemma 6.4.

We may now draw some conclusions.

Lemma 6.6. $D$ is generated in degree one. 
Proof. We need to show for $n>m$ that

$$
\Gamma\left(X, \mathcal{D}_{m, m+1}\right) \otimes_{k} \Gamma\left(X, \mathcal{D}_{m+1, n}\right) \rightarrow \Gamma\left(X, \mathcal{D}_{m, n}\right)
$$

is surjective.

The kernel of $\mathcal{D}_{m, m+1} \otimes_{X} \mathcal{D}_{m+1, n} \rightarrow \mathcal{D}_{m, n}$ has finite length whence by Lemma 6.3: $\Gamma\left(X, \mathcal{D}_{m, m+1} \otimes_{X} \mathcal{D}_{m+1, n}\right) \rightarrow \Gamma\left(X, \mathcal{D}_{m, n}\right)$ is surjective. Hence it is sufficient to show that

$$
\Gamma\left(X, \mathcal{D}_{m, m+1}\right) \otimes_{k} \Gamma\left(X, \mathcal{D}_{m+1, n}\right) \rightarrow \Gamma\left(X, \mathcal{D}_{m, m+1} \otimes_{X} \mathcal{D}_{m+1, n}\right)
$$

is surjective. In case $(r, s)=(3,2)$ we tensor $(6.2)$ for $a=0$ on the right with $\mathcal{D}_{m+1, n}$. This give

$\operatorname{Tor}_{1}^{o X}\left((0) \mathcal{D}_{m, m+1}, \mathcal{D}_{m+1, n}\right) \rightarrow(-1) \mathcal{D}_{m+1, n} \rightarrow D_{m, m+1} \otimes_{k}(0) \mathcal{D}_{m+1, n} \rightarrow(0) \mathcal{D}_{m, m+1} \otimes_{X} \mathcal{D}_{m+1, n} \rightarrow 0$

Since $\operatorname{Tor}_{1}^{o X}\left((0) \mathcal{D}_{m, m+1}, \mathcal{D}_{m+1, n}\right)$ has finite length and $(-1) \mathcal{D}_{m+1, n}$ has no finite length submodules the leftmost arrow is zero.

Hence we must show that $H^{1}\left(X,(-1) \mathcal{D}_{m+1, n}\right)=0$. This follows from Lemma 6.5.

In case $(r, s)=(2,3)$ by $(6.5)$ by a similar argument this amounts to showing that $H^{1}\left(X,(-1) \mathcal{D}_{m+1, n}\right)=0$ and $H^{1}\left(X, \mathcal{J}(0) \otimes_{X} \mathcal{D}_{m+1, n}\right)=0$. The first of these claims follows from Lemma 6.5. For the second of these claim we invoke the definition of $\mathcal{J}$ (see (6.6)). It follows that we have to show $H^{1}\left(X,(-2) \mathcal{D}_{m+1, n}\right)=0$ and $H^{2}\left(X,(-3) \mathcal{D}_{m+1, n}\right)=0$ and these are known to hold by Lemma 6.5 and Lemma 6.4 .

Our next result is that $D$ has the "correct" Hilbert function. That is

$$
\operatorname{dim} D_{m, m+a}= \begin{cases}\frac{(a+1)(a+2)}{2} & \text { if } a \geqslant 0 \\ 0 & \text { if } a<0\end{cases}
$$

The case $a<0$ is trivial so we consider $a \geqslant 0$. For this we have to check the cases $(r, s)=(3,2)$ and $(r, s)=(2,3)$ separately. For the quadratic case a computation similar to [22, Corollary 5.2.4] tells us that the colength of $\mathcal{D}_{m, m+a}$ inside $o_{X}(2 a)$ is

$$
3 \frac{a(a+1)}{2}
$$

Using the fact that $H^{1}\left(X, \mathcal{D}_{m, n}\right)=0$ by Lemma 6.5 we obtain (for $a \geqslant 0$ )

$$
\operatorname{dim} D_{m, m+a}=\frac{(2 a+1)(2 a+2)}{2}-3 \frac{a(a+1)}{2}=\frac{(a+1)(a+2)}{2}
$$

Similarly in the cubic case the colength of $\mathcal{D}_{m, m+a}$ inside $o_{X}(2 a)$ is

$$
\frac{a(a+1)}{2}
$$

and again using the fact that $H^{1}\left(X, \mathcal{D}_{m, n}\right)=0$ we obtain (for $a \geqslant 0$ )

$$
\operatorname{dim} D_{m, m+a}=\frac{(2 a+2)^{2}}{4}-\frac{a(a+1)}{2}=\frac{(a+1)(a+2)}{2}
$$

Hence in both cases (6.10) holds.

Finally we prove the following.

Lemma 6.7. The canonical map $D \rightarrow D_{Y}$ is surjective. 
Proof. As $D$ and $D_{Y}$ are both generated in degree 1 (for $D_{Y}$ this is proved in the same way as for $B(Y, \sigma, \mathcal{L})$, see [3]), it suffices to check that $D_{m, m+1} \rightarrow\left(D_{Y}\right)_{m, m+1}$ is surjective. For this consider the following commuting diagram (with $\mathcal{O}_{X}(-Y)=$ $\mathcal{O}_{X} \otimes_{o_{X}} o_{X}(-Y)$ the subobject of $\mathcal{O}_{X}$ corresonding to the ideal $g A \subset A$ )

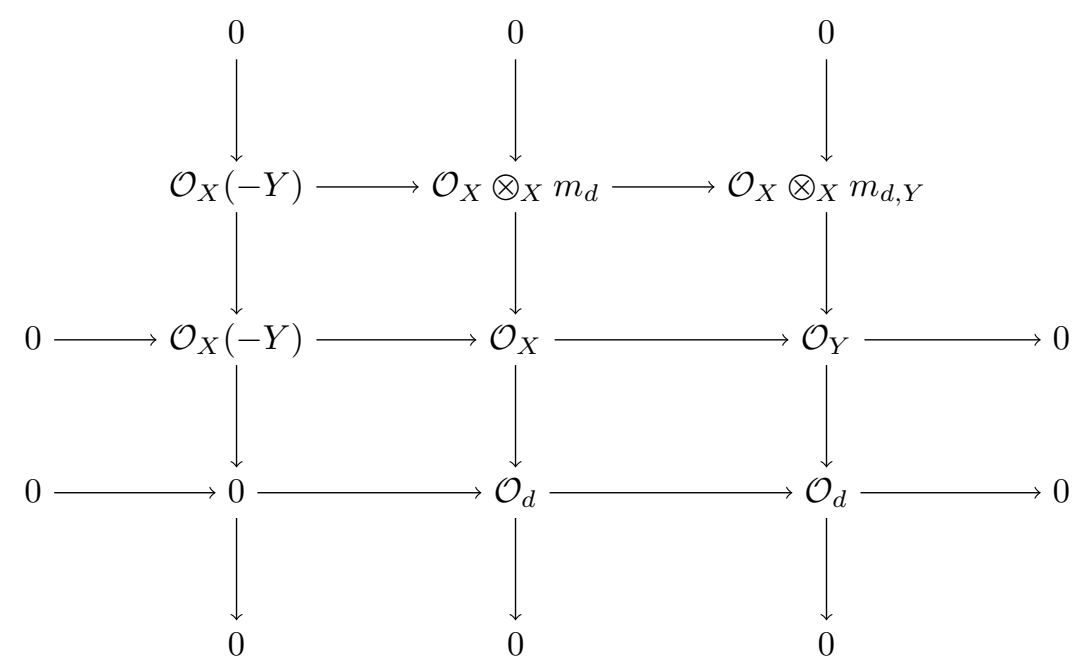

The bottom two rows and the first column are obviously exact. The third column is equal to

$$
0 \rightarrow \mathcal{M}_{d, Y} \rightarrow \mathcal{O}_{Y} \rightarrow \mathcal{O}_{d} \rightarrow 0
$$

and hence is exact. The exactness of the middle column follows as usual from [22, Lemma 8.2.1]. Hence we can apply the Snake lemma to the above diagram and find the following exact sequence:

$$
0 \rightarrow \mathcal{O}_{X}(-Y) \rightarrow \mathcal{O}_{X} \otimes_{X} m_{d} \rightarrow \mathcal{O}_{X} \otimes_{X} m_{d, Y} \rightarrow 0
$$

As the above obviously remains true when we replace $d$ by $\sigma^{-m} d$ and as $o_{X}(2)$ is an invertible bimodule we get an exact sequence

$$
0 \rightarrow \mathcal{O}_{X}(-Y) \otimes_{X} o_{X}(2) \rightarrow \mathcal{O}_{X} \otimes_{X} \mathcal{D}_{m, m+1} \rightarrow \mathcal{O}_{X} \otimes_{X}\left(\mathcal{D}_{Y}\right)_{m, m+1} \rightarrow 0
$$

The surjectivity of $D_{m, m+1} \rightarrow\left(D_{Y}\right)_{m, m+1}$ then follows from $H^{1}\left(X, \mathcal{O}_{X}(-Y) \otimes_{X}\right.$ $\left.o_{X}(2)\right)=H^{1}\left(X, \mathcal{O}_{X}(-1)\right)=0$ using that $o_{X}(-Y)=o_{X}(-3)$ (see $\left.\S 4\right)$ as well as the standard vanishing results for $X$ (see [5, Theorem 8.1]).

Since now the map $D \rightarrow D_{Y}$ is surjective, one checks using (6.10) that $D_{m, n} \rightarrow$ $D_{Y, m, n}$ is an isomorphism for $n \leqslant m+2$. Thus $D$ and $D_{Y}$ have the same quadratic relations. Let $D^{\prime}$ be the quadratic AS-regular $\mathbb{Z}$-algebra associated to $\left(Y, \mathcal{L}_{0}, \mathcal{L}_{1}\right)$ (see $\S 2$ ). Then since $D^{\prime}$ is quadratic, and has the same quadratic relations as $D_{Y}$ we obtain a surjective map $D^{\prime} \rightarrow D$. Since $D^{\prime}$ and $D$ have the same Hilbert series by (6.10) we obtain $D^{\prime} \cong D$. Hence $D$ is the quadratic AS-regular $\mathbb{Z}$-algebra associated to $\left(Y, \mathcal{L}_{0}, \mathcal{L}_{1}\right)$. 


\section{Non-COMmUtative FUnCTION FIELDS}

As above let $A$ be a 3-dimensional Sklyanin algebra, which may be either quadratic or cubic, with geometric data $(Y, \sigma, \mathcal{L})$ and let $D$ be the AS-regular $\mathbb{Z}$ subalgebra of $\check{A}^{(2)}$ constructed in $\S 4$.

Let $A^{\prime}$ be the 3 -dimensional quadratic Sklyanin algebra with geometric data $\left(Y, \sigma, \mathcal{L}_{0}\right)$ if $A$ is quadratic and $\left(Y, \psi, \mathcal{L}_{0}\right)$ if $A$ is cubic where $\left(\mathcal{L}_{i}\right)_{i}$ is as in (5.2).

By the discussion at the end of $\S 2$ together with Lemma 5.1 we conclude that $D \cong \check{A}^{\prime}$.

We will now show that there is an isomorphism between the function fields of $\operatorname{Proj} A$ and $\operatorname{Proj} A^{\prime}$. In the case that $A$ is cubic this will be the final step in the proof of Theorem 1.1. If $A$ is quadratic then the relation between $A$ and $A^{\prime}$ is a generalization of the classical Cremona transform.

By the graded version of Goldie's Theorem [11, Corollary 8.4.6.] the non-zero homogeneous elements of $A$ form an Ore set $S$ and hence the graded field of fractions $A\left[S^{-1}\right]$ of $A$ exists. By the structure theorem for graded fields [12] it is of the form

$$
\operatorname{Frac}(A)=\operatorname{Frac}_{0}(A)\left[t, t^{-1}, \alpha\right]
$$

where $\operatorname{Frac}_{0}(A)$ is a division algebra concentrated in degree zero, $|t|=1$ and $\alpha$ is an automorphism $\alpha: \operatorname{Frac}_{0}(A) \rightarrow \operatorname{Frac}_{0}(A): a \mapsto \operatorname{tat}^{-1} . \operatorname{Frac}_{0}(A)$ was introduced in the introduction as "the function field" of $\operatorname{Proj} A$. Our aim is to show that $\operatorname{Frac}_{0}(A) \cong \operatorname{Frac}_{0}\left(A^{\prime}\right)$.

It is straightforward to generalize the concept of an Ore set and its corresponding localization to $\mathbb{Z}$-algebras. In fact this is the classical concept of an Ore set in a category (and its corresponding localization).

If $S \subset A$ is a multiplicative closed Ore set consisting of homogeneous elements then one defines a corresponding multiplicative closed Ore set $\check{S} \subset \check{A}$ by putting $\check{S}_{i j}=S_{j-i}$. A straightforward check yields $\overline{A\left[S^{-1}\right]} \cong \check{A}\left[\check{S}^{-1}\right]$.

Now let $S$ and $S^{\prime}$ be the set of nonzero homogeneous elements in $A$ respectively $A^{\prime}$. Then the inclusion $\check{A}^{\prime} \hookrightarrow \check{A}^{(2)} \hookrightarrow \check{A}$ restricts to $\check{S}^{\prime} \hookrightarrow \check{S}$ and hence for arbitrary $i \in \mathbb{Z}$ there is an induced map $\zeta_{i}$ :

$\operatorname{Frac}_{0}\left(A^{\prime}\right)=\left(A^{\prime}\left[\left(S^{\prime}\right)^{-1}\right]\right)_{0} \cong\left(\check{A}^{\prime}\left[\check{S}^{\prime}-1\right]\right)_{i i} \rightarrow\left(\check{A}\left[\check{S}^{-1}\right]\right)_{2 i, 2 i} \cong\left(A\left[S^{-1}\right]\right)_{0}=\operatorname{Frac}_{0}(A)$

Although this map depends on $i$ we will show that it is always an isomorphism.

As $\operatorname{Frac}_{0}\left(A^{\prime}\right)$ and $\operatorname{Frac}_{0}(A)$ are division rings and $\zeta_{i} \neq 0$, it follows that $\zeta_{i}$ is always injective, so the only nontrivial thing to do, is proving its surjectivity. So given any $a, s \in \check{A}_{2 i, 2 j_{1}} \backslash\{0\}$ we need to find a $j_{2} \in \mathbb{Z}$ and $h \in \check{A}_{2 j_{1}, 2 j_{2}}$ such that $a h, s h \in \check{A}_{i, j_{2}}^{\prime}$. We claim we can find such an $h$ only depending on $n:=j_{1}-i$ and not on $a$ or $s$. For this consider the following map:

$$
\Gamma\left(X, o_{X}(2 n)\right) \otimes \Gamma\left(X, o_{X}(2 N) \otimes_{X} \mathcal{I}\right) \rightarrow \Gamma\left(X, o_{X}\left(2(n+N) \otimes_{X} \mathcal{I}\right)\right)
$$

where $\mathcal{I}$ is the ideal in $o_{X}$ such that $o_{X}(2(n+N)) \otimes_{X} \mathcal{I}=\mathcal{D}_{i_{1}, i_{1}+n+N}($ see $(4.5))$.

If we can choose an $N$ such that

$$
\operatorname{dim}_{k} \Gamma\left(X, o_{X}(2 N) \otimes_{X} \mathcal{I}\right) \neq\{0\}
$$

then there is an element $0 \neq h \in \check{A}_{2 i+2 n, 2 i+2 n+2 N}$ and an embedding

$$
\check{A}_{2 i, 2 i+2 n} \hookrightarrow \check{A}_{i, i+n+N}^{\prime}: a \mapsto a h
$$


which yields the surjectivity of $\operatorname{Frac}_{0}\left(A^{\prime}\right) \rightarrow \operatorname{Frac}_{0}(A)$ (as we may take $j_{2}=j_{1}+N=$ $i+n+N$ in the above). So it suffices to prove (7.1). As the cases $(r, s)=(3,2)$ and $(r, s)=(2,3)$ are completely similar, we only treat the first case.

Note that the codimension of $\Gamma\left(X, o_{X}(2 N) \otimes_{X} \mathcal{I}\right)$ inside $\check{A}_{2 i_{1}+2 n, 2 i+2 n+2 N}$ is at most $3 \frac{N(N+1)}{2}$ which grows like $\frac{3 N^{2}}{2}$. On the other hand $\operatorname{dim}_{k}\left(\check{A}_{2 i_{1}+2 n, 2 i_{1}+2 n+2 N}\right)=$ $\frac{(2 N+1)(2 N+2)}{2}$ which grows like $2 N^{2}$, so for $N$ sufficiently large (7.1) will be fulfilled.

\section{Relation With NON-COMmutative BLOWing UP}

For the interested reader we now sketch how the $\mathbb{Z}$-algebra $D$ which was introduced in a somewhat adhoc manner in $\S 4$ may be obtained in a natural way from the formalism of non-commutative blowing up as introduced in [22].

First let us remind the reader how the commutative Cremona transform works. Let $p_{1}, p_{2}, p_{3} \in \mathbb{P}^{2}$ be three distinct non-collinear points on $\mathbb{P}^{2}$ and consider the linear system of quadrics passing through those points. This is a $5-3=2$ dimensional linear system and hence it defines a birational transformation $\phi: \mathbb{P}^{2} \ldots \ldots \ldots . \mathbb{P}^{2}$ with $\left\{p_{1}, p_{2}, p_{3}\right\}$ being the points of indeterminacy.

The indeterminacy of $\phi$ may be resolved by blowing up the points $\left\{p_{1}, p_{2}, p_{3}\right\}$. Let $\alpha: \tilde{X} \rightarrow \mathbb{P}^{2}$ be the resulting surface and let $L_{1}, L_{2}, L_{3}$ be the exceptional curves. Then the Cremona transform factors as

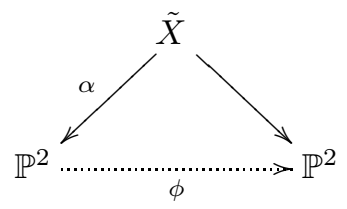

where the right most map is obtained from the sections of the line bundle $\mathcal{O}_{\tilde{X}}(1)=$ $\alpha^{*}\left(\mathcal{O}_{\mathbb{P}^{2}}(2)\right) \otimes_{\tilde{X}} \mathcal{O}_{\tilde{X}}\left(-L_{1}-L_{2}-L_{2}\right)$ on $\tilde{X}$.

Now we replace $\mathbb{P}^{2}$ by the non-commutative $X$ given by $\operatorname{Proj} A$ where $A$ is a 3 -dimensional quadratic Sklyanin algebra. We will use again the standard notation $Y, \mathcal{L}, \sigma, p_{1}, p_{2}, p_{3}, d, \ldots$ According to [22] we may blow $\mathrm{up}^{2} X$ in $d$ to obtain a map of non-commutative schemes $\alpha: \tilde{X} \rightarrow X$. Then we need a substitute for the line bundle $\mathcal{O}_{\tilde{X}}(1)$ on $\tilde{X}$. Actually in the non-commutative case it is more natural to look for a substitute for the family of objects $\left(\mathcal{O}_{\tilde{X}}(n)\right)_{n}$ since then there is an associated $\mathbb{Z}$-algebra

$$
\bigoplus_{m, n} D_{m, n}=\bigoplus_{m, n} \operatorname{Hom}_{\tilde{X}}\left(\mathcal{O}_{\tilde{X}}(-n), \mathcal{O}_{\tilde{X}}(-m)\right)
$$

This idea has been used mainly in the case that the sequence is ample in a suitable sense (e.g. [13]), but the associated $\mathbb{Z}$-algebra may be defined in general. Of course in the non-ample case the relation between the sequence and the underlying noncommutative scheme will be weaker.

Let us now carry out this program in somewhat more detail. According to [22] we have $\tilde{X}=\operatorname{Proj} \mathcal{D}$ where $\mathcal{D}$ is a graded algebra in $\operatorname{Bimod}(X-X)$ given by

$$
o_{X} \oplus m_{d}(Y) \oplus m_{d} m_{\tau^{-1} d}(2 Y) \oplus \cdots \oplus m_{d} \cdots m_{\tau^{-n+1} d}(n Y) \oplus \cdots
$$

The inclusion $o_{X} \rightarrow \mathcal{D}$ yields the map $\alpha: \tilde{X} \rightarrow X$.

\footnotetext{
${ }^{2}$ In [22] we discuss the case of a blowup of a single point. Blowing up a set of points is similar.
} 
Suitable noncommutative analogue of the objects $\mathcal{O}_{\tilde{X}}\left(-m L_{1}-m L_{2}-m L_{2}\right)$ turn out to be the objects in $\operatorname{Bimod}(X-\tilde{X})$ associated to the $o_{X}-\mathcal{D}$-bimodules given by

$m_{\tau^{m} d} \cdots m_{\tau d} \oplus m_{\tau^{m} d} \cdots m_{d}(Y) \oplus m_{\tau^{m} d} \cdots m_{\tau^{-1} d}(2 Y) \oplus \cdots \oplus m_{\tau^{m} d} \cdots m_{\tau^{-n+1} d}(n Y) \oplus \cdots$

Up to right bounded $o_{X}-\mathcal{D}$-bimodules (which are invisible in Proj) these are the same as

$$
\left(o_{X}(-m Y) \otimes_{X} \mathcal{D}\right)[m]
$$

where [1] is the shift functor on $\mathcal{D}$-modules (or bimodules, or variants thereof). So the non-commutative analogues of the objects $\mathcal{O}_{\tilde{X}}(n)$ turn out to be associated to

$$
\left(\mathcal{O}_{X}(2 n-n Y) \otimes_{X} \mathcal{D}\right)[n]
$$

where we have written $O_{X}(a+b Y)$ for $O_{X}(a) \otimes_{X} o_{X}(b Y)$.

Or ultimately

$$
\mathcal{O}_{\tilde{X}}(n)=\alpha^{*}\left(\mathcal{O}_{X}(2 n-n Y)\right)[n]
$$

We now compute (the fourth equality requires an argument similar to [22, Proposition 8.3.1(2)])

$$
\begin{aligned}
D_{m, n} & =\operatorname{Hom}_{\tilde{X}}\left(\mathcal{O}_{\tilde{X}}(-n), \mathcal{O}_{\tilde{X}}(-m)\right) \\
& =\operatorname{Hom}_{\tilde{X}}\left(\alpha^{*}\left(\mathcal{O}_{X}(-2 n+n Y)\right)[-n], \alpha^{*}\left(\mathcal{O}_{X}(-2 m+m Y)\right)[-m]\right) \\
& =\operatorname{Hom}_{X}\left(\mathcal{O}_{X}(-2 n+n Y), \alpha_{*}\left(\alpha^{*}\left(\mathcal{O}_{X}(-2 m+m Y)\right)[n-m]\right)\right) \\
& =\operatorname{Hom}_{X}\left(\mathcal{O}_{X}(-2 n+n Y), \mathcal{O}_{X}(-2 m+m Y) \otimes_{o_{X}} \mathcal{D}_{n-m}\right) \\
& \left.=\operatorname{Hom}_{X}\left(\mathcal{O}_{X}(-2 n), \mathcal{O}_{X}(-2 m) m_{\tau^{-m_{d}}} \cdots m_{\tau^{-n+1} d}\right)\right) \\
& =\Gamma\left(X, \mathcal{O}_{X}(-2 m) m_{\tau^{-m}} \cdots m_{\tau^{-n+1} d} \otimes o_{X}(2 n)\right)
\end{aligned}
$$

Hence we find indeed the same result as in $\S 4$.

\section{REFERENCES}

[1] M. Artin and W.F. Schelter. Graded algebras of global dimension 3. Adv.Math, 66:171-216, 1987.

[2] M. Artin, J. Tate, and M. Bergh. Modules over regular algebras of dimension 3. Inventiones mathematicae, 106(1):335-388, 1991.

[3] M. Artin, J. Tate, and M. Van den Bergh. Some algebras associated to automorphisms of elliptic curves. In P. et al. Cartier, editor, The Grothendieck Festschrift, volume 1 of Modern Birkhuser Classics, pages 33-85. Birkhuser Boston, 1990.

[4] M. Artin and M. Van den Bergh. Twisted homogeneous coordinate rings. Journal of Algebra, 133(2):249-271, 1990.

[5] M. Artin and J.J. Zhang. Noncommutative projective schemes. Advances in Mathematics, 109(2):228 - 287, 1994.

[6] A. Bondal and A. Polishchuk. Homological properties of associative algebras: the method of helices. Russian Acad. Sci. Izv. Math, 42:219-260, 1994.

[7] M. Brandenburg. Rosenberg's reconstruction theorem (after gabber). arXiv:1310.5978 [math.AG], 2013.

[8] D.R.Stephenson. Artin Schelter Regular algebras of global dimension three. Journal of Algebra, 183:55-73, 1996.

[9] Edgar Enochs and Sergio Estrada. Relative homological algebra in the category of quasicoherent sheaves. Adv. Math., 194(2):284-295, 2005.

[10] Pierre Gabriel. Des catégories abéliennes. Bull. Soc. Math. France, 90:323-448, 1962.

[11] C. Nastasescu and F. van Oystaeyen. Methods of Graded Rings, volume 1836 of Lecture Notes in Mathematics. Springer, 2004.

[12] Constantin Nastasescu and F. Van Oystaeyen. Graded and filtered rings and modules. Springer, Berlin, 1979 . 
[13] A. Polishchuk. Noncommutative proj and coherent algebras. Math. Res. Lett., 12(1):63-74, 2005.

[14] D. Rogalski, S.J. Sierra, and J.T. Stafford. Classifying orders in the Sklyanin algebra. arXiv:1308.2213, 2013.

[15] D. Rogalski, S.J. Sierra, and J.T. Stafford. Noncommutative blowups of elliptic algebras. Algebras and Representation Theory, pages 1-39, 2014.

[16] A. L. Rosenberg. The spectrum of abelian categories and reconstruction of schemes. In Rings, Hopf algebras, and Brauer groups (Antwerp/Brussels, 1996), pages 257-274. Dekker, New York, 1998.

[17] S. J. Sierra. G-algebras, twistings, and equivalences of graded categories. Algebr. Represent. Theory, 14(2):377-390, 2011.

[18] S.J. Sierra. Talk: Ring-theoretic blowing down (joint work with Rogalski, D. and Stafford, J.T.). Workshop Interactions between Algebraic Geometry and Noncommutative Algebra, 2014.

[19] S. P. Smith. Non-commutative Algebraic Geometry. lecture notes. University of Washington, 2000.

[20] J. T. Stafford and M. Van den Bergh. Noncommutative curves and noncommutative surfaces. Bull. Amer. Math. Soc. (N.S.), 38(2):171-216, 2001.

[21] M. Van den Bergh. A Translation Principle for the Four-Dimensional Sklyanin Algebras . Journal of Algebra, 184(2):435 - 490, 1996.

[22] M. Van den Bergh. Blowing up non-commutative smooth surfaces. Mem. Amer. Math. Soc., 154(734), 2001.

[23] M. Van den Bergh. Non-commutative quadrics. ArXiv e-prints, 2008. 